\title{
Simulating Virtual Humans Across Diverse Situations
}

\author{
Brian Mac Namee ${ }^{1}$, Simon Dobbyn $^{2}$, Pádraig Cunningham ${ }^{1} \&$ Carol O’Sullivan ${ }^{2}$ \\ ${ }^{1}$ Machine Learning Group, ${ }^{2}$ Image Synthesis Group, \\ Computer Science Department, \\ University of Dublin, Trinity College, \\ Dublin 2, \\ Ireland. \\ Brian.MacNamee@,cs.tcd.ie
}

\begin{abstract}
Perhaps due to its existentiality, the fact that simulated virtual humans give no impression of having an existence beyond their interactions with human users is often ignored in intelligent agent systems for virtual human control. This paper will describe the Proactive Persistent Agent architecture, which is designed for the control of intelligent agents in character-centric computer games. The architecture allows agents give the impression of living beyond interactions with players. Along with details of the architecture a sample simulation will be described and some steps towards evaluation will be outlined.
\end{abstract}

\section{Introduction}

In spite of appearing increasingly realistic, simulated virtual worlds (in particular those in video games) often suffer from the fact that their virtual inhabitants give no impression of having an existence beyond their interactions with human users [10]. This paper will describe an intelligent agent architecture which gives virtual humans the appearance of being involved in their own lives, even when they are not involved with a human player. This will add an extra degree of believability to simulations and allow the creation of relatively large and long term simulated environments.

In an attempt to capture the differences between the many approaches to simulating virtual humans using intelligent agents Aylett and Luck [1] present the Spectrum of Agents. One end of this spectrum focuses on physical agents which are mainly concerned with realistic physical behaviour. A notable example is the Virtual Stuntman project [6] which makes virtual actors capable of life-like motion.

Cognitive agents inhabit the other end of the agent spectrum and are mainly concerned with reasoning, decision making, planning and learning. A definitive example is Funge's cognitive modelling approach [7].

Many of the most effective systems, however, sit between these two extremes. Amongst these are $c 4$ [3], used to simulate a virtual sheep dog with the ability to learn new behaviours and the planning based Intelligent Virtual Agent system [4].

For video games, commercial solutions are also available including AI-Implant (www.ai-implant.com) which enables rule-based control of game characters and Renderware A.I. (www.renderware.com) which focuses on tactical behaviours. 
With all of these existing systems what drives the creation of yet another? The answer to this lies in the notion of virtual fidelity, as described by Badler [2]. This refers to the fact that the capabilities which should be displayed by virtual humans are determined by the applications to which they are applied. The focus of our work is on controlling Non-Player Characters (NPCs) in character-centric video games, i.e. games which focus on interactions between characters, rather than action. This positions our agents towards the cognitive end of the spectrum and leads to a specific set of fidelity requirements. Our agents are required to behave believably in a wide range of situations, possess sophisticated social ability, behave in real-time, use few resources and ease authoring for game designers. We would argue that none of the aforementioned systems satisfy this particular flavour of virtual fidelity.

This paper will proceed by detailing our architecture, and in particular the technique of role-passing. Next, a sample simulation of a university campus will be described. Finally, we will discuss evaluation, and outline directions for further work.

\section{The Proactive Persistent Agent Architecture}

In order to achieve the specific degree of virtual fidelity described above, we have created the Proactive Persistent Agent (PPA) architecture [10], where proactive refers to the fact that virtual humans pursue their own goals irrespective of the actions of human users, and persistent refers to the fact that every virtual human within a simulation is modelled (at least to some extent) at all times. The architecture has three key components - the Schedule Unit, the Role-Passing Unit and the $\mu$-SIC System (or Social Unit). These three components are connected to a shared knowledge base and to percepts which come from the simulation itself, informing a virtual human about nearby objects, events and other characters. Based on these percepts, and the contents of the knowledge base, these three units control an agent's behaviour.

In order to enable PPAs to give the impression of having a life beyond their association with simulation users, it should be possible to observe a single character as, for example, she begins her day at home, goes to work, visits a bar and, finally, returns home again. The character should behave competently in each of these situations, and in this way the illusion will be created that the character has a life beyond her involvement with simulation users.

The first step in achieving this is to equip each agent with a schedule indicating where they should be, and what they should be doing (i.e. which role they should adopt) over the course of a day of a simulation. The schedule unit achieves this.

The $\mu$-SIC system [11], is used when, as dictated by the role passing unit a character is free to socially interact with other characters, or human users. $\mu$-SIC is a connectionist system which maintains sophisticated psychological models of a character's mood and social relationships with other characters (or human users) and uses these to drive the character's social interactions.

Inspired by [8], the technique of role-passing allows agents to assume different roles over the course of a simulation. A role controls an agent's behaviour and this satisfies the requirement of believable behaviour across in diverse situations. Rolepassing begins with a basic agent, upon which different roles can be layered as 
dictated by the schedule unit. The basic agent is capable of a range of innate behaviours such as navigating the virtual world, using objects and interacting with other agents. Basic behaviours are driven by a small number of basic motivations, e.g. FEAR and HUNGER, with each motivation leading to a particular action, i.e. FLEE and EAT. These basic motivations can change both between simulations and between agents in the same simulation. For example, characters that smoke should have a basic SMOKE motivation.

PPAs are controlled using Fuzzy Cognitive Maps (FCMs) [9], which are directed graphs in which nodes represent fuzzy concepts and arcs represent fuzzy rules, or the causal flow between concepts. At each node in an FCM the activation levels of its input arcs are summed to give its activation. FCMs have been used before both to simulate entire worlds [5] and to control the behaviour of intelligent agents [12].

For our implementation, we have created an FCM system which uses a range of different fuzzy node types - concepts, motivations, rules and actions. Two sample FCMs are shown in figure 2. Node shapes indicate their types, and each arc is labelled with a '+' or a '-' to indicate whether that arc results in causal increase or decrease.

A role is simply a collection of FCM objects, and adopting a role involves adding these objects to a character's FCM. As a simulation proceeds PPAs repeatedly perform the action with the highest activation. Performing an action will result in reducing the activation of that action node, and so allows other actions to take place.

The main advantage of role passing is that it offers a simple and efficient technique for the control of agents which move competently, and believably, between situations. Not only does this does this allow believable agent behaviour, but also, by assuming and discarding roles as required, when making decisions issues unrelated to an agent's current situation are not taken into account, thus reducing the processing load.

Role-passing also makes populating a virtual world with agents a straightforward process. Placing agents within novel situations involves simply defining new roles, easing some of the complications involved in attempting to design very general agents. Furthermore designing a role involves simply drawing an FCM, and it is possible that this is more amenable to game designers than writing script or plans.

\section{Evaluation Simulation}

For demonstration purposes, a simulation of a university campus has been created. As the simulation progresses, a population of virtual humans moves between the different parts of the campus assuming and discarding a range of different roles. Implemented roles include students attending lectures, academics presenting these lectures, postgraduate students working in their offices and people relaxing in the college bar. Two screenshots of the simulation are shown in figure 1.

Figure 2 shows a basic agent (with some nodes omitted for clarity) with two of the different roles used by the simulation layered on top of it. Figure 2 (A) shows the BAR-PATRON role which is assumed when an agent is in the college bar. The important concepts for this role are THIRST, BOREDOM and SIT, the first two of which have motivations of the same name as their primary inputs, while SIT has a constant value. Each concept has attached to it an action node indicating, respectively, that the 
agent should get a drink from the bar, use some object that will entertain them (e.g. the pool table, or the dance floor) or sit down at one of the bar's tables. Connections exist between the three concept nodes with THIRST inhibiting both SIT and BOREDOM, and BOREDOM inhibiting SIT. There is also an implicit inhibition from the basic agent concepts to those in any assumed rule.
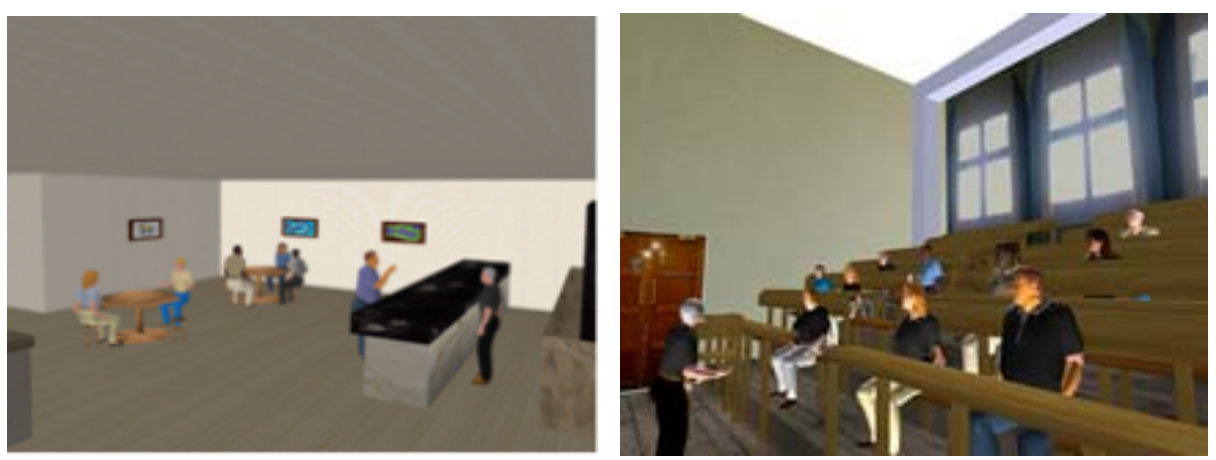

Fig. 1. Screenshots of the university simulation as implemented using the ALOHA system

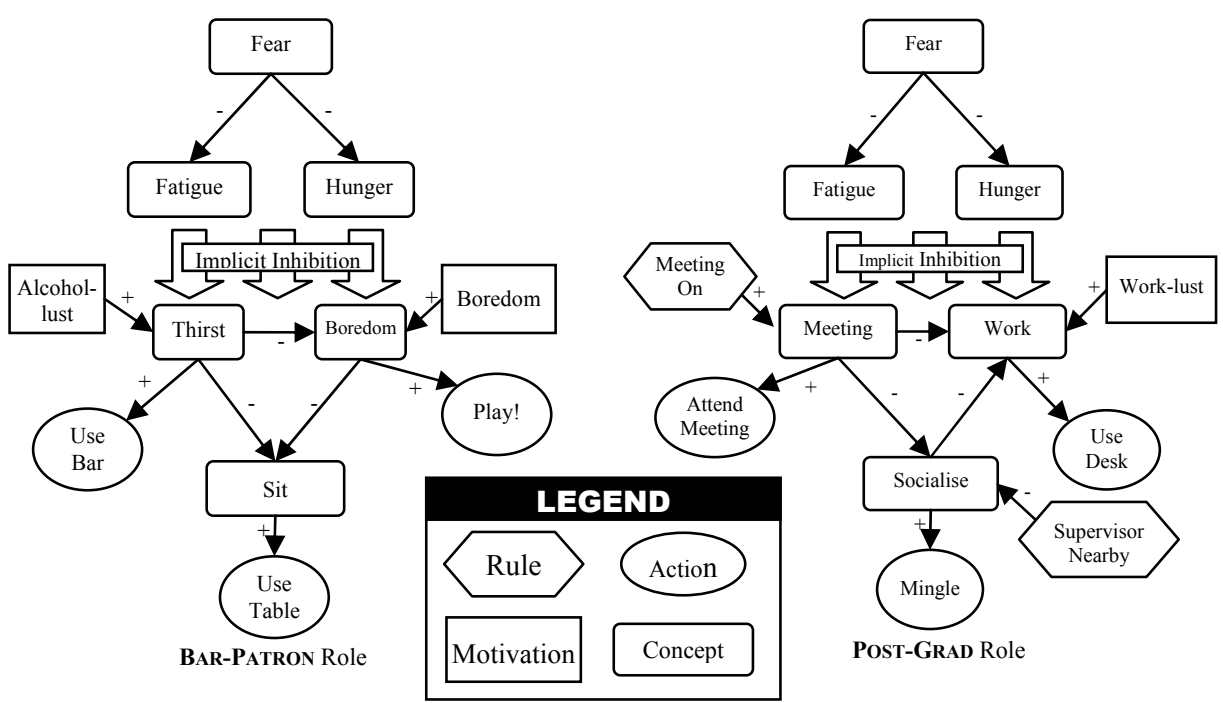

Fig. 2. Two of the roles used within the evaluation simulation, layered upon the basic agent

Figure 2 (B) shows the adoption of the POST-GRAD role used to simulate postgraduate students working in a lab. This role involves three new concepts MEETING, WORK and SOCIALISE. Activation of these concepts leads to the agent attending a meeting, performing some work at their desk or socialising, respectively. The POST-GRAD role also makes use of rule nodes with the MEETING concept increased when the MEETING-ON rule is satisfied, and the SOCIALISE concept inhibited when the SUPERVISOR-NEARBY rule is satisfied. 


\section{Conclusions}

This paper has introduced the PPA architecture and in particular the role passing technique - implemented using FCMs - which allows agents to behave believably in a range of diverse situations. This adds an extra degree of believability to simulations by populating them with virtual humans capable of creating the illusion that they carry out their lives irrespective of what the user of the simulation is doing.

The next stage in the development of the PPA architecture is to evaluate its success which will be based on the success of our simulations. Using the PPA architecture it should be possible to create a simulation of a town populated by intelligent agents. These agents should carry out their daily lives behaving believably in a wide range of situations. The levels of believability achieved by these agents should exceed that achieved by comparable modern commercial video games, for example Neverwinter Nights (nwn.bioware.com) and the Sims (thesims.ea.com). Finally, authoring the control systems for these virtual humans should be a relatively straight forward task achievable by game designers, who are usually non-programmers.

\section{References}

1. Aylett, R. \& M. Luck, Applying Artificial Intelligence To Virtual Reality: Intelligent Virtual Environments, Applied Artificial Intelligence, 14(1), 3-32, 2000.

2. Badler, N.I., R. Bindiganavale, J. Bourne, J. Allbeck, J. Shi \& M. Palmer, Real Time Virtual Humans, International Conference on Digital Media Futures, Bradford, UK, 1999.

3. Burke, R., D. Isla, M. Downie, Y. Ivanov, B. Blumberg, Creature Smarts: The Art and Architecture of a Virtual Brain, In Proc. Game Developers Conference 2001, pp. 147 - 166.

4. Caicedo, A. \& D. Thalmann, Virtual Humanoids: Let them be autonomous without losing control, The Fourth International Conference on Computer Graphics and Artificial Intelligence. Limoges, France. May 3 - 4, 2000.

5. Dickerson, J \& B Kosko, Virtual Worlds as Fuzzy Dynamical Systems, in Technology for Multimedia, B Sheu (Ed.), IEEE Press, 1996.

6. Faloutsos, P., M. van de Panne \& D. Terzopoulos, The Virtual Stuntman: Dynamic Characters with a Repertoire of Autonomous Motor Skills, Computers and Graphics, 25(6), December, 2001, 933-953.

7. Funge, J., AI for Games and Animation: A Cognitive Modeling Approach. A. K. Peters. Natick, MA, 1999.

8. Horswill, ID \& R Zubek, Robot Architectures for Believable Game Agents, Proceedings of the 1999 AAAI Spring Symposium on Artificial Intelligence and Computer Games, AAAI Technical Report SS-99-02.

9. Kosko, B, Fuzzy Engineering, Prentice Hall International, 1997.

10. Mac Namee, B \& P Cunningham, Proposal for an Agent Architecture for Proactive Persistent Non Player Characters, In D. O'Donoghue (Ed.), Proc. of the $12^{\text {th }}$ Irish Conference on AI and Cognitive Science, (pp 221 - 232), 2001.

11. Mac Namee, B \& P Cunningham, Creating Socially Interactive Non Player Characters: The $\mu$-SIC System, International Journal of Intelligent Games \& Simulations, Vol.2 No.1, February 2003.

12. Parenthoën M, P Reignier, J Tisseau, Put Fuzzy Cognitive Maps to Work in Virtual Worlds, 10th International Conference on Fuzzy Systems (Fuzz-IEEE'01), 1:252--255, Melbourne, Australia, December 2-5, 2001. 